Relations industrielles

Industrial Relations

\title{
De Coster, Michel, avec la coll. d'Annie Cornet et Christine Delhaye, Sociologie du travail et gestion du personnel
}

\section{Alain Vinet}

Volume 54, numéro 4, 1999

URI : https://id.erudit.org/iderudit/051276ar

DOI : https://doi.org/10.7202/051276ar

Aller au sommaire du numéro

Éditeur(s)

Département des relations industrielles de l'Université Laval

ISSN

0034-379X (imprimé)

1703-8138 (numérique)

Découvrir la revue

Citer ce compte rendu

Vinet, A. (1999). Compte rendu de [De Coster, Michel, avec la coll. d'Annie Cornet et Christine Delhaye, Sociologie du travail et gestion du personnel]. Relations industrielles / Industrial Relations, 54(4), 820-821.

https://doi.org/10.7202/051276ar

Tous droits réservés @ C Département des relations industrielles de l'Université Laval, 1999
Ce document est protégé par la loi sur le droit d'auteur. L’utilisation des services d'Érudit (y compris la reproduction) est assujettie à sa politique d'utilisation que vous pouvez consulter en ligne.

https://apropos.erudit.org/fr/usagers/politique-dutilisation/ 
sources humaines, on se serait attendu à ce que les activités soient systématiquement abordées selon une approche stratégique. À cet égard, les auteurs ont préféré privilégier, dans certains cas, une approche plus opérationnelle. Sur les aspects plus administratifs de la gestion des ressources humaines, certains chapitres auraient gagné à développer davantage les moyens techniques qui accompagnent l'implantation des différentes activités.

Cela dit, la plupart des chapitres sont bien documentés. La bibliographie récente atteste de l'actualité des différents propos abordés dans les chapitres et constitue une source de références supplémentaires. À l'occasion, certaines adresses de sites sur internet sont offertes au lecteur. Les maintes mises en situation et illustrations ainsi que les différents encadrés permettent à la fois de couvrir des aspects plus pratiques et de fixer les idées principales qui se dégagent du texte. Notons également que deux ou trois cas terminent chacun des chapitres. Ces cas, qui sont succincts, constituent une source d'apprentissage appréciée des étudiants puisqu'ils leur permettent d'appliquer les notions apprises dans le chapitre. Aussi, cet ouvrage nous fait bénéficier des travaux de recherche des auteurs qui y ont collaboré.

En somme, ce livre demeure un ouvrage de base intéressant qui examine avec originalité la gestion des ressources humaines. Il permet de faire le point sur les principales activités et illustre astucieusement un domaine qui, à certains égards, peut paraître très normatif, soit celui de la gestion des ressources humaines.

\section{TANIA SABA \\ École de relations industrielles Université de Montréal}

\section{Sociologie du travail et gestion des ressources humaines}

par Michel DE COSTER avec la collaboration d'Annie CORNET et de Christine

DELHAYE, Bruxelles : DeBoeck Université, 1999, 3édition [1987], 344 p., ISBN

2-8041-3122-X.

Il s'agit de la troisième édition d'un ouvrage fort apprécié paru en 1987 sous le titre Sociologie du travail et gestion $d u$ personnel. Cette troisième édition est significativement revue et augmentée compte tenu du contexte de l'activité socio-économique (chômage chronique, aménagement du temps de travail, informatisation des organisations) et du désir de l'auteur de rendre son ouvrage accessible à un public plus étendu que celui des étudiants et des chercheurs, en s'adressant notamment aux gestionnaires des ressources humaines dans les entreprises.

Cet ouvrage n'est pas qu'un livre d'initiation parmi d'autres. Il se distingue des autres à la fois par son intention et par son écriture. L'intention est originale: éclairer la gestion des ressources humaines par la perspective sociologique du travail pour mieux l'ancrer dans la réa- lité sociale des organisations. Pour De Coster, il est urgent de faire comprendre les enjeux réels des techniques de gestion trop souvent " plaquées sur des tissus sociaux dont on n'a retenu ni la diversité ni l'originalité ". C'est précisément parce que la gestion des ressources humaines présente un caractère instrumental et normatif qu'il lui est interdit de faire l'économie de la description et de l'observation :

"Avant d'agir sur la réalité organisationnelle, il convient de la connaître, à travers sa culture, ses valeurs, ses traditions, la configuration de ses groupes sociaux réputés formels ou informels, ses jeux et ses enjeux de pouvoir. Au surplus, méconnaître l'analyse sociologique consacrerait dans la réalité des faits, l'échec fréquent des techniques de gestion greffées sur des tissus sociaux très divers. Pour avoir méconnu cette idée capitale 
qu'une technique de gestion des ressources humaines est tributaire de particularismes socioculturels, des managers, animés des meilleures intentions au demeurant, ont vu leurs savantes combinaisons techniques s'écrouler comme châteaux de cartes. Ils avaient pourtant devant les yeux les leçons de l'histoire moderne et notamment celle de la coopération occidentale avec les pays en voie de développement "(p. 16).

Voilà qui donne le ton de l'ouvrage : rédigé avec verve et agilité, dans un langage accessible aux non-initiés, utilisant des exemples et des formules qui font réfléchir.

Visiblement, l'auteur est un expert dans le maniement de l'écriture, le choix d'une image mentale qui fait passer le message, la vulgarisation scientifique. Cette qualité d'écriture et le souci pédagogique qui l'anime contribuent tout autant que l'intention à distinguer cet ouvrage parmi d'autres livres d'initiation.

L'ouvrage est divisé en deux grandes parties. La première partie est consacrée aux éléments de la sociologie du travail qui ancrent la gestion des ressources humaines dans son contexte social. C'est, à mon avis, la meilleure partie du livre: elle comporte des chapitres très bien articulés sur le travail comme valeur sociale, sur la division du travail, sur la technologie et sur la logique du pouvoir dans les organisations. Bref, l'auteur y atteint son objectif en offrant au lecteur (étudiant, gestionnaire) des outils pour comprendre le milieu dans lequel il est ou il deviendra un acteur.

La deuxième partie qui porte sur la gestion des ressources humaines ne présente pas la même cohérence: les chapitres sont plus disparates et les liens moins évidents. La perspective est aussi plus hésitante entre la sociologie des organisations, la théorie des organisations et la pratique du management. De plus, l'auteur admet d'emblée qu'il ne peut couvrir tout le champ de la GRH. Il fait cependant des choix qui s'avèrent très judicieux : cette partie comprend notam. ment un chapitre sur la rémunération et un chapitre sur l'aménagement du temps de travail où les implications majeures et les enjeux socio-économiques de ces deux volets de la gestion du travail sont explicités. J'ai trouvé plus sommaires les chapitres sur les politiques de GRH et sur la gestion de la qualité.

Un livre d'initiation n'introduit pas de grande nouveauté et celui-ci ne fait pas exception.

Son attrait provient plutôt de ce qu'il incite le lecteur à prendre le temps de la réflexion, à ne pas sauter aux conclusions, à comprendre les problèmes actuels dans leur enracinement historique et à envisager toutes les conséquences de l'implantation de nouvelles techniques de gestion.

Enfin, De Coster offre à ses lecteurs une synthèse des connaissances actuelles agréable à lire et rehaussée de l'expérience acquise durant de nombreuses années d'enseignement. Cette synthèse est appuyée sur une documentation abondante et actualisée, principalement européenne et francophone. Les exemples et les illustrations proviennent surtout de France et de Belgique et à l'occasion des États-Unis et du Japon.

ALAIN VINET

Département des relations industrielles Université Laval

\section{Négociation en relations du travail : nouvelles approches \\ sous la direction de Pierre DESCHÊNES, Jean-Guy BERGERON, Reynald BOURQUE et André BRIAND, Sainte-Foy : Presses de l'Université du Québec, 1998, 183 p., ISBN 2-7605-0920-6.}

Voici un ouvrage collectif qui s'inscrit dans ce mouvement d'implantation d'une culture de concertation patronalesyndicale dans les entreprises québécoi- 